\title{
Using multiple cytokines to predict hepatocellular carcinoma recurrence in two patient cohorts
}

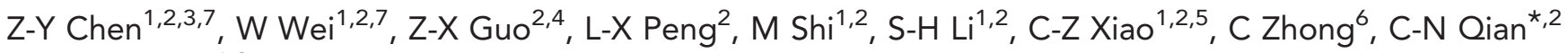 \\ and R-P Guo*,1,2
}

${ }^{1}$ Department of Hepatobiliary Surgery, Sun Yat-sen University Cancer Center, 651 Dongfeng Road East, Guangzhou 510060, China; ${ }^{2}$ Sun Yat-sen University Cancer Center, State Key Laboratory of Oncology in South China, Collaborative Innovation Center for Cancer Medicine, 651 Dongfeng East Road, Guangzhou 510060, China; ${ }^{3}$ Department of Gastrointestinal Surgery, the Sixth Affiliated Hospital of Sun Yat-sen University, Guangzhou 510655, China; ${ }^{4}$ Department of Ultrasound, Sun Yat-Sen University Cancer Center, 651 Dongfeng Road East, Guangzhou 510060, China; ${ }^{5}$ Department of General surgery, Shenzhen Shajing Affiliated Hospital of Guangzhou Medical University, Shengzheng 518104, Guangdong, China and ${ }^{6}$ Department of Hepatobiliary Surgery, 1st Affiliated Hospital to Guangzhou University of Chinese Medicine, 16 Jichang Road, Guangzhou 510405, China

Background: Cytokines are tightly linked to the carcinogenesis, development and prognosis of hepatocellular carcinoma (HCC). We determined the prognostic value of 39 circulating cytokines in $\mathrm{HCC}$ patients after radical resection and then developed a novel cytokine-based prognostic classifier (CBPC) for the prediction of patient prognosis.

Methods: A total of 179 patients were divided into two cohorts based on the date of radical resection. Thirty-nine cytokines were simultaneously analysed in patient serum samples using multiplex bead-based Luminex technology. Support vector machinebased methods and Cox proportional hazard models were used to develop a CBPC from the training cohort, which was then validated in the validation cohort.

Results: Among seven cytokines significantly correlating with the disease-free survival (DFS) in the training cohort, six of them were validated to be significant prognostic factors to predict DFS and overall survival (OS) in the validation cohort, namely fibroblast growth factor 2 (FGF-2), growth-regulated oncogene (GRO), interleukin 8 (IL-8), interferon gamma-induced protein 10 (IP-10), vascular endothelial growth factor (VEGF), and interferon alpha-2 (IFN- $\alpha 2)$. By integrating six cytokines and three clinical characteristics, we developed a CBPC to predict the recurrence and 3-year OS of HCC patients (sensitivity, 0.648; specificity, 0.918). In the validation cohort, the CBPC were confirmed to have significant predictive power for predicting tumour recurrence and OS (sensitivity, 0.585; specificity, 0.857). Interestingly, IFN- $\alpha 2$ was the only cytokine being independent prognostic factor in both patient cohorts.

Conclusion: Our study verifies the presence of specific cytokine-phenotype associations with patient prognosis in HCC. The CBPC developed include multiple circulating cytokines and may serve as a novel screening approach for identifying HCC patients with a high risk of post-resection recurrence and shorter OS. These individuals may also be suitable for cytokine-targeted therapies.

Hepatocellular carcinoma (HCC) is characterised by highly vascularised and rapid tumour progression, a high recurrence rate after surgical resection, and an extremely poor prognosis
(Bruix and Llovet, 2009; Ayyappan and Jhaveri, 2010; Chen et al, 2011). Hepatocellular carcinoma is the fifth most common cancer in the world, and the third most frequent cause of cancer death

\footnotetext{
${ }^{\star}$ Correspondence: Professor C-N Qian; E-mail: qianchn@sysucc.org.cn or Professor R-P Guo; E-mail: guorp@sysucc.org.cn ${ }^{7}$ These authors contributed equally to this work.
} 
(Jemal et al, 2011; Li et al, 2012; Chen et al, 2013). Chronic infection of the hepatitis B or $\mathrm{C}$ virus, together with the consequent immune response, has an important role in the carcinogenesis and development of HCC (Luan et al, 2009; An et al, 2010; Arzumanyan et al, 2013).

Cytokines have traditionally been viewed as attractants for inflammatory leucocytes. However, accumulating evidence suggest that cytokines and their receptors act as key regulators of the tumour microenvironment and have a role in many pathological entities, including chronic hepatitis B and cirrhotic liver disease. In addition, evidence suggests that cytokines are involved in carcinogenetic processes, such as autonomous growth signalling, which influence tumour growth, invasion and metastasis (Vingerhoets et al, 1998; Chan and Sung, 2006; Capone et al, 2010). Cytokines are secreted by a variety of cell types, including fibroblasts, endothelial cells, epithelial cells, macrophages, and cancer cells. It has been reported that some host-derived cytokines can suppress tumour formation by controlling infection, inflammation, and immunity (Cahlin et al, 2000; Balkwill, 2004). It has also been reported that tumour cells secrete and exploit hostderived cytokine that such are crucial for the formation of tumour stroma and blood vessel networks, which are processes that can lead to therapeutic resistance and poor prognosis (Lurje et al, 2008; Mantovani et al, 2008; Niu et al, 2008; Wu et al, 2010, 2011).

In the present study, we used multiplex bead-based Luminex technology to detect 39 circulating cytokines in serum samples collected from two cohorts of HCC patients who underwent R0 resection. From these data, we developed a predictive cytokinebased prognostic classifier (CBPC).

\section{MATERIALS AND METHODS}

Patient selection. This study enroled 179 HCC patients (median age, 56 years; range, 26-68 years) who were hospitalised at the Sun Yat-sen University Cancer Center between January 2006 and December 2010. Patients were selected based on the following criteria: (1) a history of histopathologically confirmed HCC and (2) the use of curative liver resection as primary HCC therapy. Curative resection (R0 resection) was defined as macroscopically complete removal of the tumour with a resection margin that was histopathologically negative of tumour (Foster, 1984). Microvascular invasion was defined as microscopic tumour invasion indentified in portal or hepatic vein of the surrounding liver tissue, which was contiguous to the tumour (Toyosaka et al, 1996). Based on the time of enrolment, the first 103 cases were assigned to the training cohort and the remaining 76 cases were assigned to the validation cohort. Preoperative serum samples were collected from all patients (Figure 1). The study was approved by the Ethics Committee of Sun Yat-sen University Cancer Center, and informed consent was obtained from all patients. Only patients who survived more than 60 days post surgery were included in the study. All patients had an Eastern Cooperative Oncology Group (ECOG) status of two or lower and a Child-Pugh classification of A.

Patients were followed postoperatively with regular surveillance for recurrence using the serum alpha-fetoprotein (AFP) level, abdominal ultrasonography, and computed tomography. Followup information was obtained in December 2012 from patient medical records and telephone correspondence with patients and their families. The median time of follow-up is 20.73 month (range, 3-78 months). The median disease-free survival (DFS) was 20.63 months (range, 2-52 months) for all patients. There were 99 (55.3\%) case of recurrent HCC and 67 deaths (37.4\%). Disease-free survival was defined as the interval between surgery and recurrence or between surgery and censoring without recurrence. Overall

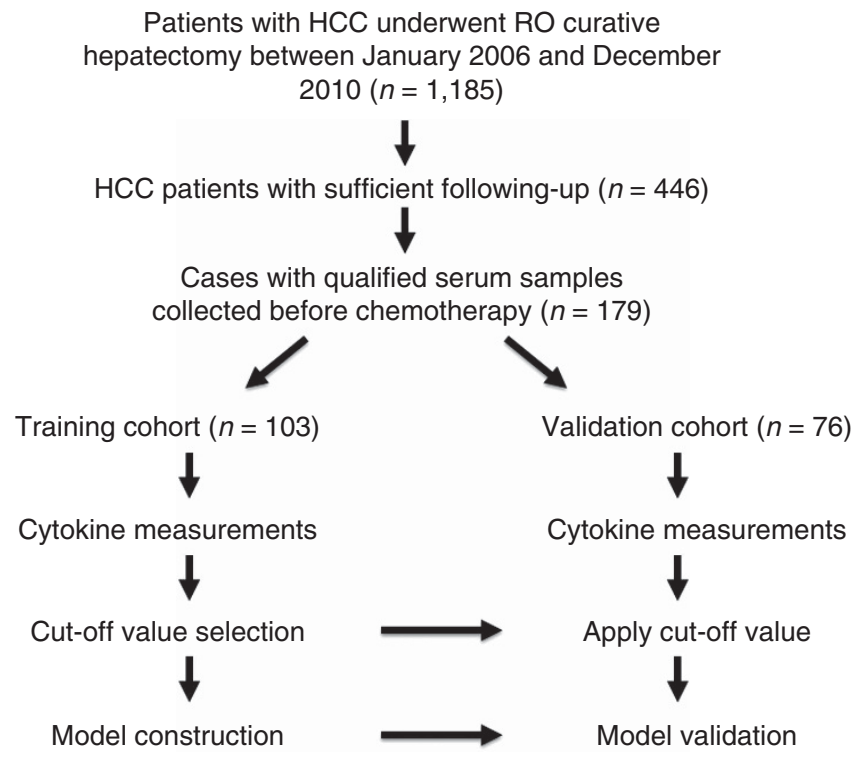

Figure 1. A schematic overview of patient selection. The schematic summarises the cases for construction and validation of the classifier.

survival (OS) was defined as the interval between surgery and death or between surgery and censoring.

Cytokine measurements. Peripheral blood was collected by a standard protocol. Briefly, blood samples were allowed to clot at room temperature for $30-90 \mathrm{~min}$, followed by centrifugation at $2000 \mathrm{~g}$ for $10 \mathrm{~min}$. The serum samples were then aliquoted and stored at $-80^{\circ} \mathrm{C}$.

The concentrations of 39 cytokines were analysed in duplicate using Human Cytokine Antibody-Immobilized Magnetic Beads (Millipore, Billerica, MA, USA). The cytokines analysed were as follows: interleukin-1 alpha (IL-1 $\alpha$ ), IL-1 $\beta$, IL-1RA, IL-2, IL-3, IL-4, IL-5, IL-6, IL-7, IL-8, IL-9, IL-10, IL-13, IL-15, IL-17, IL-12P40, IL-12P70, epidermal growth factor (EGF), eotaxin, granulocyte colony-stimulating factor (G-CSF), granulocyte macrophage colony-stimulating factor (GM-CSF), interferon alpha-2 (IFN- $\alpha 2$ ), IFN $\gamma$, interferon gamma-induced protein 10 (IP-10), monocyte chemotactic protein-1 (MCP-1), MCP-3, MIP- $1 \alpha$, MIP- $1 \beta$, tumour necrosis factor alpha (TNF $\alpha), \operatorname{TNF} \beta$, vascular endothelial growth factor (VEGF), fibroblast growth factor 2 (FGF-2), transforming growth factor alpha (TGF- $\alpha$ ), Flt-3 ligand (FIT-3L), fractalkine, growth-regulated oncogene (GRO), macrophage-derived chemokine (MDC), soluble CD40 ligand (sCD40L), and soluble interleukin-2 receptor alpha (sIL-2Ra). Measurements were performed on a Luminex 200 analyzer (Luminex, Austin, TX, USA).

Statistical analyses. All statistical analyses were performed using SPSS version 16.0 software (SPSS Inc, Chicago, IL, USA). Survival rates were assessed by life tables. Kaplan-Meier analyses and logrank test were used to compare the difference in survival rates. Cox proportional hazard models were used for survival analyses. A twotailed $P$-value less than 0.05 was considered statistically significant.

Selection of cut-off values for Kaplan-Meier survival analyses. Cut-off values were selected based on receiver operating characteristic (ROC) curve analyses using SPSS version 16.0 software (Hanley, 1989; Spira and Ettinger, 2004; Zlobec et al, 2007). To generate ROC curves for the detected values of each cytokine, the sensitivity and specificity of a recurrence event were plotted in the training cohort. The value closest to the point of maximum sensitivity and specificity (i.e., the point $(0.0,1.0)$ on the curve) was selected as the cut-off score that correctly classified the largest 
number of patients with or without a recurrence event. Each cut-off value was then tested in the validation cohort. The cytokines were dichotomized in ROC curve analyses.

Table 1. A detailed description of the construction of CBPC in the training cohort

\begin{tabular}{|l|c|c|c|c|}
\hline & Value & Patient, $\boldsymbol{n}$ & 2-year DFS (\%) & 3-year OS (\%) \\
\hline CBPC & $\leqslant 33.45$ & 64 & 48.4 & 31.25 \\
\hline & $>33.45$ & 39 & 5.1 & 5.1 \\
\hline
\end{tabular}

Abbreviations: $\mathrm{CBPC}=$ cytokine-based prognostic classifier; DFS $=$ disease-free survival; FGF-2 = fibroblast growth factor $2 ; G R O=$ growth-regulated oncogene; IFN- $\alpha 2=$ interferon alpha-2; IL-8 = interleukin $8 ; \mathrm{IP}-10=$ interferon gamma-induced protein $10 ; \mathrm{OS}=$ overall survival; $T N M=$ tumour node metastasis; $V E G F=$ vascular endothelial growth factor. $\mathrm{CBPC}=(\mathrm{FGF}-2 \times 1.7+\mathrm{IFN}-\alpha 2 \times 7.5+\mathrm{GRO} \times 2.4+\mathrm{IL}-8 \times 0.9+\mathrm{IP}-10 \times 1.4+\mathrm{VEGF} \times 1.9+$

tumour diameter $\times 1.9+$ tumour multiplicity $\times 2.1+$ TNM stage $\times 1.4)$.
Prognostic classifier construction. The clinical characteristics and the cytokines found to be significant in the univariate analyses were entered into a Cox proportional hazards model. A multiple linear regression model was constructed using the variable that multiplied their respective hazard ratios in a Cox proportional hazards model: $\left(Y=X_{1} \times \mathrm{HR}_{1}+X_{2} \times \mathrm{HR}_{2}+\ldots+X \mathrm{n} \times \mathrm{HRn}\right)$. The sum of variables (e.g., $Y$ ) was used to construct an ROC curve depicting the relationship between the sensitivity and specificity for a recurrence event. The performance of the classifier was tested by the prediction accuracy in validation cohort (Table 1).

\section{RESULTS}

Univariate analyses on clinicopathological factors. Except for tumour diameter, there was no significant difference in the

Table 2. Clinicopathological characteristics of the patients with tumour diameter, tumour multiplicity and TNM stage being significant prognostic factors for DFS in both cohorts (Kaplan-Meier method)

\begin{tabular}{|c|c|c|c|c|c|c|}
\hline \multirow[b]{2}{*}{ Characteristics } & \multicolumn{3}{|c|}{ Training cohort } & \multicolumn{3}{|c|}{ Validation cohort } \\
\hline & Patients, $n$ & 2-year DFS rate (\%) & $\boldsymbol{P}$-value & Patients, $n$ & 2-year DFS rate (\%) & $P$-value \\
\hline Gender & & & 0.605 & & & 0.810 \\
\hline Male & 94 & 33.0 & & 66 & 39.4 & \\
\hline Female & 9 & 22.2 & & 10 & 40.0 & \\
\hline Age & & & 0.876 & & & 0.509 \\
\hline$<50$ & 51 & 32.9 & & 42 & 40.5 & \\
\hline$\leqslant 50$ & 52 & 25.0 & & 34 & 38.2 & \\
\hline HBsAg & & & 0.139 & & & 0.464 \\
\hline Positive & 93 & 30.1 & & 65 & 41.5 & \\
\hline Negative & 10 & 50.0 & & 11 & 27.3 & \\
\hline Cirrhosis & & & 0.204 & & & 0.093 \\
\hline No & 32 & 46.9 & & 21 & 52.4 & \\
\hline Yes & 71 & 25.4 & & 55 & 34.5 & \\
\hline Tumour encapsulation & & & 0.565 & & & 0.100 \\
\hline Present & 75 & 34.7 & & 61 & 45.9 & \\
\hline Absent & 28 & 25.0 & & 15 & 13.3 & \\
\hline Tumour diameter & & & $<0.001$ & & & 0.002 \\
\hline$\leqslant 5 \mathrm{~cm}$ & 50 & 50.0 & & 21 & 66.7 & \\
\hline$>5 \mathrm{~cm}$ & 53 & 15.1 & & 55 & 29.1 & \\
\hline Tumour multiplicity & & & 0.001 & & & $<0.001$ \\
\hline Solitary & 73 & 38.4 & & 49 & 53.1 & \\
\hline Multiple & 30 & 16.7 & & 27 & 14.8 & \\
\hline Histological grade & & & 0.078 & & & 0.252 \\
\hline Well differentiated & 61 & 41.0 & & 44 & 43.2 & \\
\hline Poorly differentiated & 42 & 19.0 & & 32 & 34.4 & \\
\hline AFP & & & 0.236 & & & 0.025 \\
\hline$\leqslant 25$ ug & 44 & 36.4 & & 22 & 59.1 & \\
\hline$>25$ ug & 59 & 28.8 & & 54 & 50.0 & \\
\hline Microvascular invasion & & & 0.466 & & & 0.036 \\
\hline Present & 12 & 8.3 & & 7 & 14.3 & \\
\hline Absent & 91 & 35.2 & & 69 & 42.0 & \\
\hline TNM stage & & & 0.010 & & & $<0.001$ \\
\hline$|-| \mid$ & 58 & 39.7 & & 45 & 57.8 & \\
\hline III-IV & 45 & 22.2 & & 31 & 12.9 & \\
\hline
\end{tabular}


demographic or clinical characteristics between the training and the validation cohorts. As expected, using univariate analyses, tumour diameter, tumour multiplicity, and tumour node metastasis (TNM) stage were identified as significant prognostic factors affecting DFS in the training and validation cohorts (Table 2). Tumour diameter and tumour multiplicity were also identified as significant risk factors affecting OS.

Cytokine features associated with recurrence. Among the 39 cytokines analysed, EGF, EOTAXIN, GRO, MDC, IP-10, MCP-1, and VEGF were found in all HCC patient serum samples collected before radical resection. In contrast, IL-3 and sCD40L were not detected in any HCC patient serum samples. The ROC curves for some features clearly illustrate the point on the curve closest to 0.0 , 1.0, which maximises both sensitivity and specificity for the outcome (Figure 2). Univariate analysis of the training cohort indicated that increased levels of five cytokines (FGF-2, GRO, IL-8, IP-10, and VEGF) were associated with poor DFS, whereas increased levels of two cytokines (IFN- $\alpha 2$ and MIP1 $\beta$ ) were associated with longer disease-free survival. Among these seven cytokines, six of them were confirmed to be significant prognostic factors in the validation cohort, namely FGF-2, GRO, IL-8, IP-10, VEGF, and IFN- $\alpha 2$ (Table 3).

Multivariate analysis of the training cohort indicated that IFN- $\alpha 2$ ( $P \leqslant 0.001$; HR, 7.486), GRO $(P=0.016$; HR, 2.426), and tumour multiplicity $(P=0.019 ; \mathrm{HR}, 2.117)$ retained their importance for predicting DFS. Multivariate analysis of the validation cohort indicated that IFN- $\alpha 2(P=0.008$; HR, 2.561), IL-8 $(P=0.015 ; H R, 2.505)$, and tumour diameter $(P=0.002$; $\mathrm{HR}, 4.554)$ were independent prognostic indicators of DFS. Therefore, only IFN- $\alpha 2$ was the only cytokine being independent favourable prognostic factor in both patient cohorts (Table 4).

Construction of the classifier. We next integrated three clinicopathologic characteristics (tumour diameter, tumour multiplicity, and TNM stage) with six cytokines (FGF-2, GRO, IL-8, IP-10, VEGF, and IFN- $\alpha 2$ ) into CBPC to construct a classifier for the prediction of HCC recurrence on the training cohort. The classification accuracy for recurrence after curative liver resection was 0.783 (sensitivity, 0.648; specificity, 0.918). The Kaplan-Meier curve also showed that this classifier could effectively separate high-risk (2-year DFS rates, 48.4\%) patients from low-risk patients (2-year DFS rate, 5.1\%) (Figure 3).

When analysis was tested on the validation cohort using the CBPC, 29 patients were identified as high risk and 47 as low risk. The 2 -year DFS rates were $10.3 \%$ for the high-risk patients and $57.4 \%$ for the low-risk patients in the validation cohort. The CBPC was also strongly predictive of OS in the validation cohort (sensitivity, 0.585; specificity, 0.857). The 3-year OS rate was $20.7 \%$ for the high-risk patients and $61.7 \%$ for the low-risk patients in this cohort (Figure 4A and B). When all patients were analysed as a combined cohort, the CBPC classified 68 patients as high risk and 111 patients as low risk. The 2-year DFS was $7.4 \%$ for the high-risk patients and $52.3 \%$ for the low-risk patients in this group. The CBPC was also strongly predictive of OS when all patients were treated as a combined cohort (sensitivity, 0.642; specificity, 0.777 ). The 3 -year OS rate was $11.8 \%$ for the high-risk patients and $44.1 \%$ for the low-risk patients in this group (Figure $4 \mathrm{C}$ and D).

The correlation between clinical characteristics and CBPC was also evaluated by $\chi^{2}$ analysis in the combined cohort. No significant correlation was found with regard to other clinicopathological factor except histological grade in the combined cohort. The patients in the low-risk group usually had well differentiated HCC, while the patient in the high-risk group with poorly differentiated HCC.
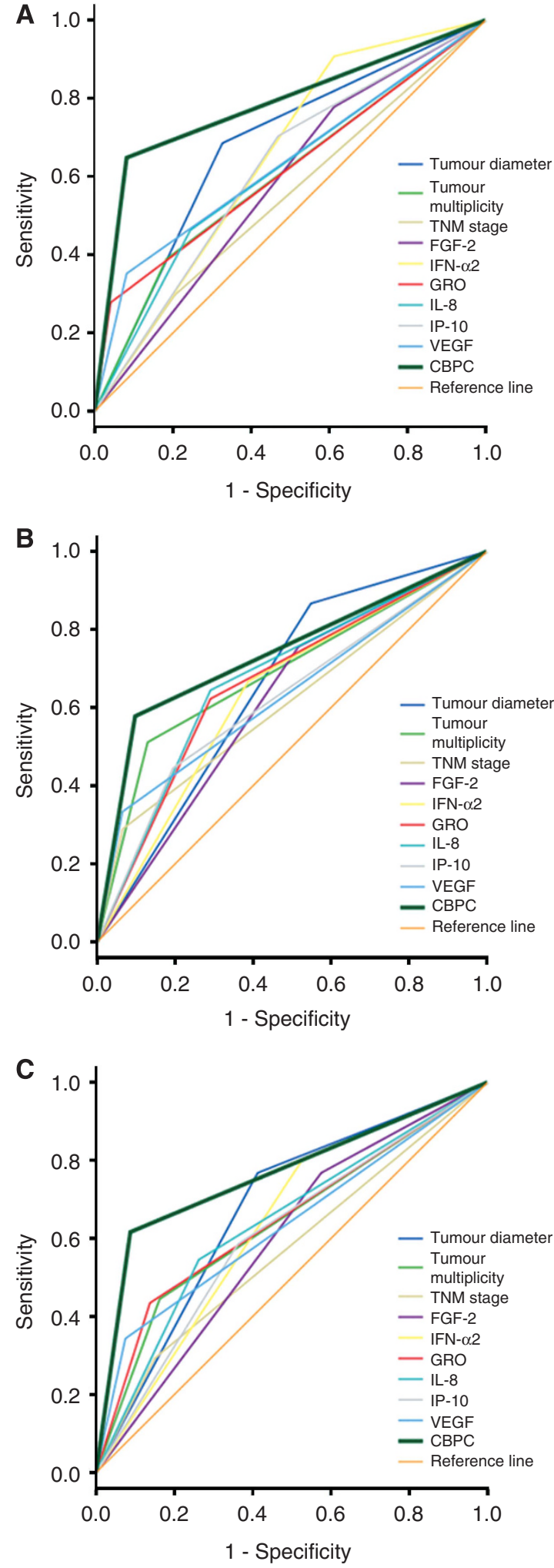

Figure 2. Receiver operating characteristic curves. These curves are of the significant clinicopathological factors, six cytokines and the CBPC predicting recurrent event using the training cohort $(\mathbf{A})$, the validation cohort $(\mathbf{B})$ and the combine cohort $(\mathbf{C})$ that includes both the training and validation cohorts. Cytokine-based prognostic classifier showed the largest value of the sensitivity multiplying the specificity when compared with other factor. 
Table 3. Among the seven cytokines significantly correlating with DFS in the training cohort, six of them were consistently validated in the validation cohort

Training cohort

Validation cohort

\begin{tabular}{|c|c|c|c|c|c|c|}
\hline Cytokine (pg ml $\left.{ }^{-1}\right)$ & Patients, $n$ & $\begin{array}{c}\text { 2-year DFS } \\
\text { rate }(\%)\end{array}$ & $\boldsymbol{P}$-value & Patients, $n$ & $\begin{array}{c}\text { 2-year DFS } \\
\text { rate }(\%)\end{array}$ & $\boldsymbol{P}$-value \\
\hline FGF-2 & & & 0.037 & & & 0.026 \\
\hline$\leqslant 9.66$ & 31 & 54.8 & & 26 & 53.8 & \\
\hline$>9.66$ & 72 & 22.2 & & 50 & 32.0 & \\
\hline IFN- $\alpha 2$ & & & 0.001 & & & 0.014 \\
\hline$\leqslant 4.425$ & 79 & 24.1 & & 42 & 26.2 & \\
\hline$>4.425$ & 24 & 58.3 & & 34 & 55.9 & \\
\hline GRO & & & $<0.001$ & & & 0.018 \\
\hline$\leqslant 548.5$ & 86 & 37.2 & & 39 & 53.8 & \\
\hline$\geqslant 548.5$ & 17 & 5.9 & & 37 & 24.3 & \\
\hline IL-8 & & & 0.004 & & & 0.002 \\
\hline$\leqslant 18.93$ & 66 & 42.4 & & 38 & 57.9 & \\
\hline$>18.93$ & 37 & 13.5 & & 38 & 21.1 & \\
\hline IP-10 & & & 0.012 & & & 0.014 \\
\hline$\leqslant 223.5$ & 42 & 50.0 & & 50 & 48.0 & \\
\hline$>223.5$ & 61 & 19.7 & & 26 & 23.1 & \\
\hline MIP1 $\beta$ & & & 0.022 & & & 0.520 \\
\hline$\leqslant 25.35$ & 64 & 25.0 & & 46 & 41.3 & \\
\hline$>25.35$ & 39 & 43.6 & & 30 & 36.7 & \\
\hline VEGF & & & 0.001 & & & 0.004 \\
\hline$\leqslant 151$ & 80 & 37.5 & & 59 & 49.2 & \\
\hline$>151$ & 23 & 13.0 & & 17 & 5.9 & \\
\hline
\end{tabular}

Abbreviations: DFS = disease-free survival; FGF-2 = fibroblast growth factor 2; GRO = growth-regulated oncogene; IFN- $\alpha 2=$ interferon alpha-2; IL-8 = interleukin 8 ; IP-10 = interferon gammainduced protein 10; VEGF = vascular endothelial growth factor.

Table 4. Multivariate analyses of the risk factors for DFS in HCC patients

Training cohort

Validation cohort

\begin{tabular}{|l|c|c|c|c|c|c|}
\cline { 2 - 7 } & $\boldsymbol{P}$-value & HR & $\mathbf{9 5 . 0 \%} \mathbf{C l}$ & $\boldsymbol{P}$-value & $\mathbf{H R}$ & $\mathbf{9 5 . 0 \%} \mathrm{Cl}$ \\
\hline FGF-2 & 0.199 & 1.670 & $0.764-3.650$ & 0.162 & 1.665 & $0.814-3.402$ \\
\hline IFN- $\alpha 2$ & $<0.001$ & 7.486 & $2.820-19.871$ & 0.008 & 2.561 & $1.283-5.114$ \\
\hline GRO & 0.016 & 2.426 & $1.176-5.006$ & 0.292 & 1.452 & $0.726-2.907$ \\
\hline IL-8 & 0.834 & 0.928 & $0.462-1.864$ & 0.015 & 2.505 & $1.198-5.240$ \\
\hline IP-10 & 0.063 & 1.878 & $0.967-3.644$ & 0.096 & 1.698 & $0.911-3.166$ \\
\hline VEGF & 0.354 & 1.397 & $0.689-2.835$ & 0.171 & 1.637 & $0.808-3.315$ \\
\hline Tumour diameter & 0.073 & 1.947 & $0.939-4.038$ & 0.002 & 4.554 & $1.759-11.787$ \\
\hline Tumour multiplicity & 0.019 & 2.117 & $1.129-3.972$ & 0.102 & 2.100 & $0.863-5.113$ \\
\hline TNM stage & 0.348 & 1.406 & $0.690-2.862$ & 0.604 & 0.765 & $0.287-2.104$ \\
\hline
\end{tabular}

Abbreviations: $\mathrm{Cl}=$ confidence interval; $\mathrm{DFS}=$ disease-free survival; $\mathrm{FGF}-2=$ fibroblast growth factor $2 ; \mathrm{GRO}=$ growth-regulated oncogene; $\mathrm{HR}=$ hazard ratio; IFN- $\alpha 2=$ interferon alpha-2; $\mathrm{IL}-8=$ interleukin 8 ; IP-10 = interferon gamma-induced protein 10; TNM = tumour node metastasis; VEGF = vascular endothelial growth factor.

\section{DISCUSSION}

Accumulating evidence strongly suggest that cancer cells in different organs can secrete distinct profiles of cytokines to promote growth and metastasis, as well as to manipulate host tumour interactions (Balkwill, 2004; Gourzones et al, 2012).
Classification of patients by cytokine profiles has successfully improved the prognosis of several malignancies (Seruga et al, 2008). Because cytokines expression has been shown to be heterogeneously distributed within the tumours, the detection of serum level cytokines will likely be a more reliable approach for measuring the general expression of tumour-originated cytokines. Moreover, for host-originated cytokines, detection of cytokines at 

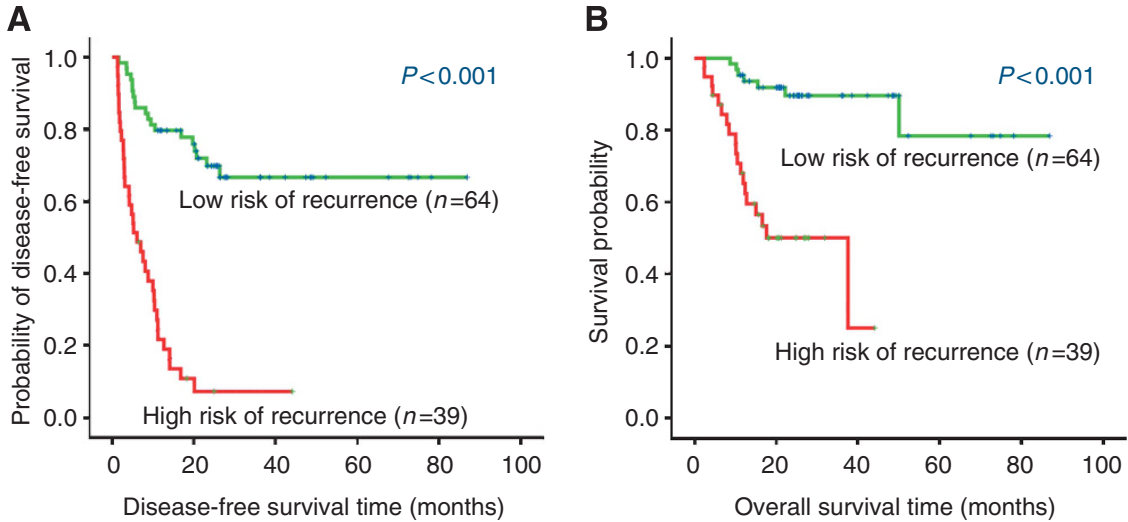

Figure 3. The survival curves of HCC patients in the training cohort. (A) Disease-free survival. (B) Overall survival. Significant differences were found in the both comparisons between high- and low-risk groups.
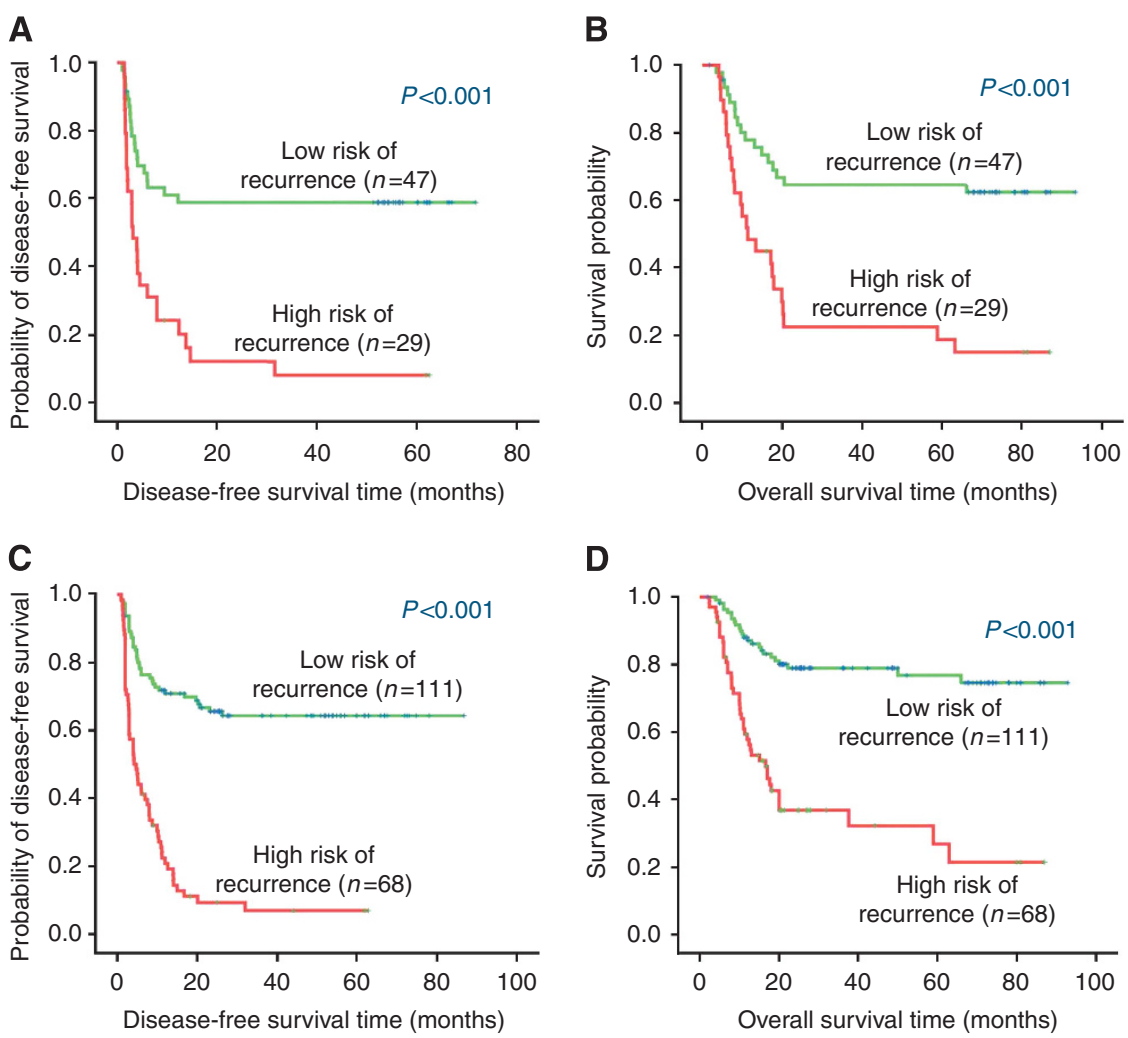

Figure 4. Application of CBPC to refine the assessment of risk in HCC patients. Kaplan-Meier survival estimates for HCC patients with a high or low probability of recurrence in the validation cohort $(\mathbf{A})$ and the combine cohort $(\mathbf{C})$. The survival curves of HCC patients with a high or low probability of death in the validation cohort (B), and the combine cohort (D). Significant differences were found in all of the comparisons between the high- and low-risk groups.

the serum level is the more reliable approach. However, the broad investigation of cytokine networks is largely limited by the low throughput of conventional ELISA methods, which typically allow for testing of only one cytokine at a time (Levina et al, 2008). Using a high-sensitivity human cytokine detection kit, we successfully detected multiple cytokines in the serum of HCC patients. Our findings demonstrate a spectrum of cytokines expression in HCC. Among the 39 cytokines simultaneously evaluated, 6 cytokines (FGF-2, GRO, IL-8, IP-10, VEGF, and IFN- $\alpha 2$ ) were associated with DFS.

Staging systems have been widely used to classify patients into different risk groups and to guide treatment. Although a number of HCC staging systems (Okuda stage, TNM stage, BCLC staging, CLIP score, JIS score, and CUPI score) have been proposed, none have been universally accepted (Lu et al, 2008; Hsu et al, 2010; Huitzil-Melendez et al, 2010; op den Winkel et al, 2012). Our proposed CBPC, which integrates clinicopathologic features with significantly expressed cytokines, demonstrated high prognostic predictive power for the HCC patients who underwent radical resection. The prognostic classifiers in our study provided a new strategy and approach for making optimal clinical decisions for patients with HCC. Adjuvant transcatheter arterial chemoembolisation (TACE) and targeted therapy (such as sorafenib) had moderate efficacy with serious 
side effects. With our established prognostic tool, clinicians can select the adjuvant treatment that would maximise the therapeutic benefit and minimise the risk of overtreatment for patient with high risk of recurrence.

Compared with other sophisticated SVM models, our CBPC enables rapid and practical HCC prognostic predictions by a clinician, without the need of special software or expertise (Zhu et al, 2009; Xu et al, 2012). The serum samples used to train and validate our CBPC were collected from patients with normal liver function before R0 resection. Moreover, there was no correlation between CBPC and other clinical characteristics except histological grade. Therefore, the CBPC can be applied to patients with good performance status who have undergone other radical treatments, such as liver transplantation or local ablation therapy.

Previous studies have shown that tumour cells take advantage of the expression of cytokines and cytokine receptors to stimulate an immune response and to induce tumour angiogenesis and tumour growth, process that promote metastasis. Blocking the signalling pathways of key cytokines (FGF-2, GRO, IL-8, and VEGF) can reduce tumour growth and metastasis (Wang et al, 2006; Llovet et al, 2008; Chen et al, 2012; Ning and Lenz, 2012). Modulation of immune responses by directly targeting cytokines or cytokine genes (siRNA, neutralising antibody and small-molecule inhibitors) is an emerging anti-cancer strategy. Our study demonstrates that IFN- $\alpha 2$ was favourably correlated with DFS in HCC patients in both cohorts. In contrast, elevated expression of FGF-2, GRO, IL-8, IP-10, and VEGF are correlated with shorter DFS in HCC patients. Based on the results of this study, we conclude that blocking a single cytokine may be ineffective, inhibitors to target multiple cytokines may improve HCC prognosis. Additional clinical studies are warranted to explore the therapeutic value of inhibiting or activating multiple cytokine signalling pathways in HCC.

In conclusion, our study suggests that both cytokine levels in serum and cytokine-based classifiers can better predict the prognosis of HCC patients. The technically simple and reliable cytokine measurements used in this study can facilitate identification of the high-risk HCC patients who may benefit from novel cytokine-targeted therapies.

\section{ACKNOWLEDGEMENTS}

This work was supported by grants from the National Natural Science Foundation of China (No. 81030043, No. 81272340, and No. $81172037 / \mathrm{H} 1606)$.

\section{CONFLICT OF INTEREST}

The authors declare no conflict of interest.

\section{AUTHOR CONTRIBUTIONS}

Study concept and design: Rong-Ping Guo and Chao-Nan Qian. Acquisition of data: Zhi-Yuan Chen, Zhi-Xing Guo, Li-Xia Peng, and Cheng-Zuo Xiao. Analysis and interpretation of data: ZhiYuan Chen, Wei Wei, Shu-Hong Li, Chong Zhong, and Chao-Nan Qian. Drafting of the manuscript: Zhi-Yuan Chen and Chao-Nan Qian. Critical revision of the manuscript for important intellectual content: Wei Wei and Ming Shi. Statistical analysis: Zhi-Yuan Chen, Wei Wei and Chao-Nan Qian. Obtained funding: RongPing Guo and Chao-Nan Qian.

\section{REFERENCES}

An HJ, Jang JW, Bae SH, Choi JY, Cho SH, Yoon SK, Han JY, Lee KH, Kim DG, Jung ES (2010) Sustained low hepatitis B viral load predicts good outcome after curative resection in patients with hepatocellular carcinoma. J Gastroenterol Hepatol 25(12): 1876-1882.

Arzumanyan A, Helena MG, Reis HM, Feitelson MA (2013) Pathogenic mechanisms in HBV- and HCV-associated hepatocellular carcinoma. Nat Rev Cancer 13(2): 123-135.

Ayyappan AP, Jhaveri KS (2010) CT and MRI of hepatocellular carcinoma: an update. Expert Rev Anticancer Ther 10(4): 507-519.

Balkwill F (2004) Cancer and the chemokine network. Nat Rev Cancer 4(7): 540-550.

Bruix J, Llovet JM (2009) Major achievements in hepatocellular carcinoma. Lancet 373(9664): 614-616.

Cahlin C, Korner A, Axelsson H, Wang W, Lundholm K, Svanberg E (2000) Experimental cancer cachexia: the role of host-derived cytokines interleukin (IL)-6, IL-12, interferon-gamma, and tumor necrosis factor alpha evaluated in gene knockout, tumor-bearing mice on $\mathrm{C} 57 \mathrm{Bl}$ background and eicosanoid-dependent cachexia. Cancer Res 60(19): 5488-5493.

Capone F, Costantini S, Guerriero E, Calemma R, Napolitano M, Scala S, Izzo F, Castello G (2010) Serum cytokine levels in patients with hepatocellular carcinoma. Eur Cytokine Netw 21(2): 99-104.

Chan HL, Sung JJ (2006) Hepatocellular carcinoma and hepatitis B virus. Semin Liver Dis 26(2): 153-161.

Chen WQ, Zheng RS, Zhang SW (2013) Liver cancer incidence and mortality in China, 2009. Chin J Cancer 32(4): 162-169.

Chen ZY, Shi M, Peng LX, Wei W, Li XJ, Guo ZX, Li SH, Zhong C, Qian CN, Guo RP (2012) Dovitinib preferentially targets endothelial cells rather than cancer cells for the inhibition of hepatocellular carcinoma growth and metastasis. J Transl Med 10: 245.

Chen ZY, Wei W, Guo ZX, Lin JR, Shi M, Guo RP (2011) Morphologic classification of microvessels in hepatocellular carcinoma is associated with the prognosis after resection. J Gastroenterol Hepatol 26(5): $866-874$.

Foster JH (1984) Computing operative risk. Surgery 95(5): 631.

Gourzones C, Barjon C, Busson P (2012) Host-tumor interactions in nasopharyngeal carcinomas. Semin Cancer Biol 22(2): $127-136$.

Hanley JA (1989) Receiver operating characteristic (ROC) methodology: the state of the art. Crit Rev Diagn Imaging 29(3): 307-335.

Hsu CY, Hsia CY, Huang YH, Su CW, Lin HC, Lee PC, Loong CC, Chiang JH, Huo TI, Lee SD (2010) Selecting an optimal staging system for hepatocellular carcinoma: comparison of 5 currently used prognostic models. Cancer 116(12): 3006-3014.

Huitzil-Melendez FD, Capanu M, O’Reilly EM, Duffy A, Gansukh B, Saltz LL, Abou-Alfa GK (2010) Advanced hepatocellular carcinoma: which staging systems best predict prognosis? J Clin Oncol 28(17): 2889-2895.

Jemal A, Bray F, Center MM, Ferlay J, Ward E, Forman D (2011) Global cancer statistics. CA Cancer J Clin 61(2): 69-90.

Levina V, Su Y, Nolen B, Liu X, Gordin Y, Lee M, Lokshin A, Gorelik E (2008) Chemotherapeutic drugs and human tumor cells cytokine network. Int J Cancer 123(9): 2031-2040.

Li XP, Cao GW, Sun Q, Yang C, Yan B, Zhang MY, Fu YF, Yang LM (2012) Cancer incidence and patient survival rates among residents in the Pudong New Area of Shanghai between 2002 and 2006. Chin J Cancer 32(9): 512-519.

Llovet JM, Ricci S, Mazzaferro V, Hilgard P, Gane E, Blanc JF, de Oliveira AC, Santoro A, Raoul JL, Forner A, Schwartz M, Porta C, Zeuzem S, Bolondi L, Greten TF, Galle PR, Seitz JF, Borbath I, Haussinger D, Giannaris T, Shan M, Moscovici M, Voliotis D, Bruix J (2008) Sorafenib in advanced hepatocellular carcinoma. $N$ Engl J Med 359(4): 378-390.

Lu W, Dong J, Huang Z, Guo D, Liu Y, Shi S (2008) Comparison of four current staging systems for Chinese patients with hepatocellular carcinoma undergoing curative resection: Okuda, CLIP, TNM and CUPI. J Gastroenterol Hepatol 23(12): 1874-1878.

Luan F, Liu H, Gao L, Liu J, Sun Z, Ju Y, Hou N, Guo C, Liang X, Zhang L, Sun W, Ma C (2009) Hepatitis B virus protein preS2 potentially promotes HCC development via its transcriptional activation of hTERT. Gut 58(11): 1528-1537. 
Lurje G, Zhang W, Schultheis AM, Yang D, Groshen S, Hendifar AE, Husain H, Gordon MA, Nagashima F, Chang HM, Lenz HJ (2008) Polymorphisms in VEGF and IL-8 predict tumor recurrence in stage III colon cancer. Ann Oncol 19(10): 1734-1741.

Mantovani A, Allavena P, Sica A, Balkwill F (2008) Cancer-related inflammation. Nature 454(7203): 436-444.

Ning Y, Lenz HJ (2012) Targeting IL-8 in colorectal cancer. Expert Opi Ther Targets; e-pub ahead of print 12 April 2012.

Niu J, Azfer A, Zhelyabovska O, Fatma S, Kolattukudy PE (2008) Monocyte chemotactic protein (MCP)-1 promotes angiogenesis via a novel transcription factor, MCP-1-induced protein (MCPIP). J Biol Chem 283(21): 14542-14551.

op den Winkel M, Nagel D, Sappl J, op den Winkel P, Lamerz R, Zech CJ, Straub G, Nickel T, Rentsch M, Stieber P, Goke B, Kolligs FT (2012) Prognosis of patients with hepatocellular carcinoma. Validation and ranking of established staging-systems in a large western HCC-cohort. PLoS One 7(10): e45066.

Seruga B, Zhang H, Bernstein LJ, Tannock IF (2008) Cytokines and their relationship to the symptoms and outcome of cancer. Nat Rev Cancer 8(11): 887-899.

Spira A, Ettinger DS (2004) Multidisciplinary management of lung cancer. N Engl J Med 350(4): 379-392.

Toyosaka A, Okamoto E, Mitsunobu M, Oriyama T, Nakao N, Miura K (1996) Intrahepatic metastases in hepatocellular carcinoma: evidence for spread via the portal vein as an efferent vessel. Am J Gastroenterol 91(8): $1610-1615$.

Vingerhoets J, Michielsen P, Vanham G, Bosmans E, Paulij W, Ramon A, Pelckmans P, Kestens L, Leroux-Roels G (1998) HBV-specific lymphoproliferative and cytokine responses in patients with chronic hepatitis B. J Hepatol 28(1): 8-16.
Wang B, Hendricks DT, Wamunyokoli F, Parker MI (2006) A growth-related oncogene/CXC chemokine receptor 2 autocrine loop contributes to cellular proliferation in esophageal cancer. Cancer Res 66(6): 3071-3077.

Wu MH, Hong HC, Hong TM, Chiang WF, Jin YT, Chen YL (2011) Targeting galectin-1 in carcinoma-associated fibroblasts inhibits oral squamous cell carcinoma metastasis by downregulating MCP-1/CCL2 expression. Clin Cancer Res 17(6): 1306-1316.

Wu WK, Llewellyn OP, Bates DO, Nicholson LB, Dick AD (2010) IL-10 regulation of macrophage VEGF production is dependent on macrophage polarisation and hypoxia. Immunobiology 215(9-10): 796-803.

Xu J, Ding T, He Q, Yu XJ, Wu WC, Jia WH, Yun JP, Zhang Y, Shi M, Shao CK, Pan WD, Yin XY, Min J, Zhuang SM, Zheng L (2012) An in situ molecular signature to predict early recurrence in hepatitis B virus-related hepatocellular carcinoma. J Hepatol 57(2): 313-321.

Zhu ZH, Sun BY, Ma Y, Shao JY, Long H, Zhang X, Fu JH, Zhang LJ, Su XD, Wu QL, Ling P, Chen M, Xie ZM, Hu Y, Rong TH (2009) Three immunomarker support vector machines-based prognostic classifiers for stage IB non-small-cell lung cancer. J Clin Oncol 27(7): 1091-1099.

Zlobec I, Steele R, Terracciano L, Jass JR, Lugli A (2007) Selecting immunohistochemical cut-off scores for novel biomarkers of progression and survival in colorectal cancer. J Clin Pathol 60(10): $1112-1116$.

This work is published under the standard license to publish agreement. After 12 months the work will become freely available and the license terms will switch to a Creative Commons AttributionNonCommercial-Share Alike 3.0 Unported License. 